\title{
Electronic and optical properties of amorphous GaSe thin films
}

\author{
M. C. Siqueira' ${ }^{1}$ K. D. Machado $^{2}$ J. P. M. Serbena ${ }^{2}$ I. A. Hümmelgen ${ }^{2}$. \\ S. F. Stolf ${ }^{3}$ C. G. G. de Azevedo ${ }^{4}$ - J. H. D. da Silva ${ }^{4}$
}

Received: 15 February 2016/Accepted: 18 March 2016/Published online: 1 April 2016

(C) Springer Science+Business Media New York 2016

\begin{abstract}
The eletronic and optical properties of amorphous GaSe thin films produced by vacuum evaporation were investigated using X-ray photoemission spectroscopy (XPS) and transmittance spectroscopy techniques. XPS measurements allowed the determination of the valence band energy and showed the chemical bonding and the charge transfer between Se and Ga atoms. Transmittance measurements allowed the determination of the optical gap, refractive index and extinction coefficient in the low and high absorption regions. Using the Wemple and DiDomenico single oscillator model we also found the oscillator and the dispersive energies. From the valence band and optical gap energies, the conduction band was found and an energy level diagram for $f$-GaSe is proposed.
\end{abstract}

\section{Introduction}

Chalcogenide alloys formed by elements of the III-VI families have attracted much attention in recent years due to their possible technological applications. Selenium is the element with the highest work function [1] and for this reason it is very attractive for electronic and optoelectronic

K. D. Machado

kleber@fisica.ufpr.br

1 Faculdade de Tecnologia SENAI-CIC, R. Senador Accioly Filho, 298, CIC, Curitiba, PR 81310-000, Brazil

2 Departamento de Física, Centro Politécnico, Universidade Federal do Paraná, Curitiba, PR 81531-990, Brazil

3 Centro de Engenharia e Ciências Exatas, UNIOESTE, Toledo, PR 85903-000, Brazil

4 Departamento de Física, Universidade Estadual Paulista, Bauru, SP 17033-360, Brazil demands regarding hole transport. Despite this attractive characteristic, Se films sublimate due to the high vapor pressure even at room temperature, turning difficult its practical use. In this context, PSe thin films were recently investigated, also showing high work function and applicability as hole injection electrode in organic semiconductor based devices [2]. Despite showing a suppression of the sublimation problem, this PSe alloy presents long term instability attributed to the chemical reactivity of $\mathrm{P}$ in the presence of oxygen and water rich atmosphere. The substitution of $\mathrm{P}$ still preserving the low vapor pressure of the alloy, but increasing the chemical stability of the material would be highly desirable when aiming at device applications. A possible candidate is $\mathrm{Ga}$, since $\mathrm{Ga}-\mathrm{Se}$ alloys have very interesting properties, such as wide spectral transparency, ranging from 0.65 to $18 \mu \mathrm{m}$ [3], high nonlinear coefficients [4,5] and large optical gaps [6-13].

Recently some of us investigated an amorphous alloy of the Ga-Se system, with the composition $\mathrm{GaSe}_{9}\left(a-\mathrm{GaSe}_{9}\right)$, produced by MA, regarding its structural [14], thermal and photothermal $[14,15]$ properties. However, to develop possible applications of $\mathrm{Ga}-\mathrm{Se}$ alloys and of $\mathrm{GaSe}_{9}$ in particular, it is important to investigate their properties in the form of thin films. Thus, in this study, we produced amorphous $\mathrm{GaSe}$ thin films ( $f$-GaSe) by the vacuum evaporation (VE) technique using the powders of $a-\mathrm{GaSe}_{9}$ produced by MA, and we investigated their electronic and optical properties.

To investigate the GaSe thin films, we used x-ray photoemission spectroscopy (XPS) and transmittance spectroscopy techniques. XPS measurements allow us to show the existence of chemical bonding between $\mathrm{Se}$ and $\mathrm{Ga}$ at the atomic level. The shifts in the Se and Ga levels indicate a charge transfer from $\mathrm{Ga}$ atoms to Se atoms. In addition, XPS data also show the absence of contamination by other 
chemical species and they allow determining the valence band energy $\left(E_{v b}\right)$ of $f$-GaSe. From the transmittance measurements, we obtain the optical gap $E_{g}$ using the McLean procedure [16]. We also use a routine based on the Cisneros method [17] to obtain the refractive index $n$ and the extinction coefficient $k$ in the low absorption region. With these values, and using the Wemple and DiDomenico model [18] we extrapolate $n$ for the high absorption region, and calculate $k$ using the extrapolated $n$ and the measured transmittances. We also found the values of the oscillator $\left(E_{0}\right)$, dispersive $\left(E_{d}\right)$ energies, static refractive index $(n(0))$ and static dieletric constant $(\varepsilon(0))$. With the optical gap and the valence band energy we determine the conduction band energy $E_{c b}$, and propose an energy level diagram for $f$ $\mathrm{GaSe}_{9}$, which is an important information in developing devices based on $\mathrm{GaSe}_{9}$.

\section{Theoretical background}

\subsection{Optical gap determination}

The most suitable method to extract the optical gap is to determine the wavelength where a linear extrapolation of the absorption edge crosses the zero absorption abscissa. However, the linearity is obtained in different plots. According to electronic structure theory [16, 19-21], the absorption of different semiconductors shall follow the equation:

$\alpha h v=B\left(h v-E_{g}\right)^{1 / m}$,

where $\alpha$ is the absorption coefficient, $B$ is a constant and $E_{g}$ is the gap energy and $v$ is the frequency of the incident radiation, and $m$ is an integer related to the nature of the electronic transitions in the materials. In crystalline semiconductors, direct allowed transitions corresponds to $m=2$, forbidden direct transitions correspond to $m=\frac{2}{3}$, allowed indirect transitions correspond to $m=\frac{1}{2}$, while $m=\frac{1}{3}$ holds for forbidden indirect transitions $[16,19,20]$. Besides, in disordered semiconductors, spite of the different theoretical development as compared to crystalline materials [21, 22], Eq. 1 is often observed for $m=\frac{1}{2}$, and the corresponding bandgap determination is known as Tauc procedure [21]. Equation 1 will be used to determine the bandgap and for the analysis of the absorption edges of films.

\subsection{Refractive index and extinction coefficient}

The refractive index, extinction coefficient and film thickness were obtained from the transmittance data, using a computational routine based on the Cisneros method [17].
The method is based on the complete solutions of Maxwell equations for homogeneous absorbing films onto transparent thick substrates. The maxima and minima of the transmittance in the transparent and weakly absorbing regions at energies below the band gap are used in the routine to determine the refractive index dispersion and film thickness. The routine uses the values of the transmittance in the extrema to determine the (small) extinction coefficient iteratively and to refine the refractive index values calculated using the transparent film formulae [23]. The set of refined refractive index values corresponding to the energies of the transmittance extrema are used in the Wemple and DiDomenico [18] single-oscillator model to calculate the dispersion of the refractive index, according to the interpolation formula

$$
n^{2}(E)=1+\frac{E_{0} E_{d}}{E_{0}^{2}-E^{2}},
$$

where the parameters $E_{0}$ and $E_{d}$ are the oscillator and the dispersive energies, respectively, and $E=h v$ is the photon energy. The refractive index obtained using this method is extrapolated to the absorption edge region, in order to determine the values of the absorption coefficient, and the extinction coefficient $k$ in this region. The absorption coefficient was determined using the optical transmittance spectra and the extrapolated refractive index and thickness determined in the weak absorption region. The method is based on the exponential dependence of the transmittance on the absorption coefficient in an interactive procedure. The rapidly converging calculations assure very precise determination of the absorption coefficient [17].

\section{Experimental details}

Thin films of GaSe ( $f$-GaSe) with an estimated thickness of $800 \mathrm{~nm}$ were produced by evaporation at $10^{-5}$ mbar of the milled powders of $a$ - $\mathrm{GaSe}_{9}$ prepared according to the procedure described on Ref. [15] using a W-crucible. The depositions were performed onto $n$-Si, for XPS measurements, and quartz, for transmittance measurements. For XPS measurements we also made thin films of pure Se $(f$-Se) deposited on $n$-Si following the same procedure described above.

XPS measurements were made at room temperature at the D04A-SXS beamline of the Brazilian Synchrotron Light Laboratory (LNLS). The base pressure was around $1 \times 10^{-9}$ mbar. The photon energy was set to $1840 \mathrm{eV}$ and the corresponding energy resolution was about $0.4 \mathrm{eV}$. The mean probing depth of XPS at this photon energy was approximately $35 \AA$ [24]. The spectra were normalized to the maximum and the energy scale was calibrated using a clean gold foil. 
Optical transmittance spectra at normal incidence in the 200-3300 nm range were obtained using a Perkin Elmer Lambda 1050 double beam spectrophotometer.

\section{Results and discussion}

\subsection{XPS measurements}

Figure 1 shows a typical XPS spectrum of $f$-GaSe (black lines) and, for comparison, the XPS spectrum of $f$-Se (green line) both deposited on $n$-Si. From Fig. 1, the selenium levels in $f-\mathrm{GaSe}_{9}$ are found at $55 \mathrm{eV}$, for $3 d$ level, at 161 and $167 \mathrm{eV}$ for $3 p_{3 / 2}$ and $3 p_{1 / 2}$ levels, respectively, and at $230 \mathrm{eV}$ for $3 s$ level. For comparison, in $f$-Se these levels are found at 56 ( $3 d$ level), 162 and $167 \mathrm{eV}\left(3 p_{3 / 2}\right.$ and $3 p_{1 / 2}$ levels), and $231 \mathrm{eV}$ ( $3 s$ level). In the case of gallium peaks, the $3 d$ level is located at $20.4 \mathrm{eV}$ and the $3 p_{3 / 2}$ and $3 p_{1 / 2}$ levels are found at 106 and $109 \mathrm{eV}$, respectively. These levels are found, in crystalline Ga [25], at $18.5 \mathrm{eV}\left(3 d\right.$ level) and at 103.5 and $106.4 \mathrm{eV}\left(3 p_{3 / 2}\right.$ and $3 p_{1 / 2}$ levels). Thus, in $f$-GaSe, Se levels are shifted to lower energies, and $\mathrm{Ga}$ levels are shifted to higher energies. These features show the existence of chemical bonds between Se and $\mathrm{Ga}$ atoms. In addition, they indicate that electrons of $\mathrm{Ga}$ atoms are transferred to Se atoms.

The inset of Fig. 1 presents the valence band photoemission of $f$-GaSe, which is mainly composed of Se $4 s$ and $4 p$ and $\mathrm{Ga} 4 s$ and $4 p$ states. The top of the valence band (indicated by the dashed red line) appears at a binding energy around $1.44 \pm 0.06 \mathrm{eV}$. Considering this value and the cut-off of the photoemission spectra $\left(E_{c}=4.4 \mathrm{eV}\right)$, the valence band was estimated at $E_{v b}=5.84 \pm 0.06 \mathrm{eV}$.

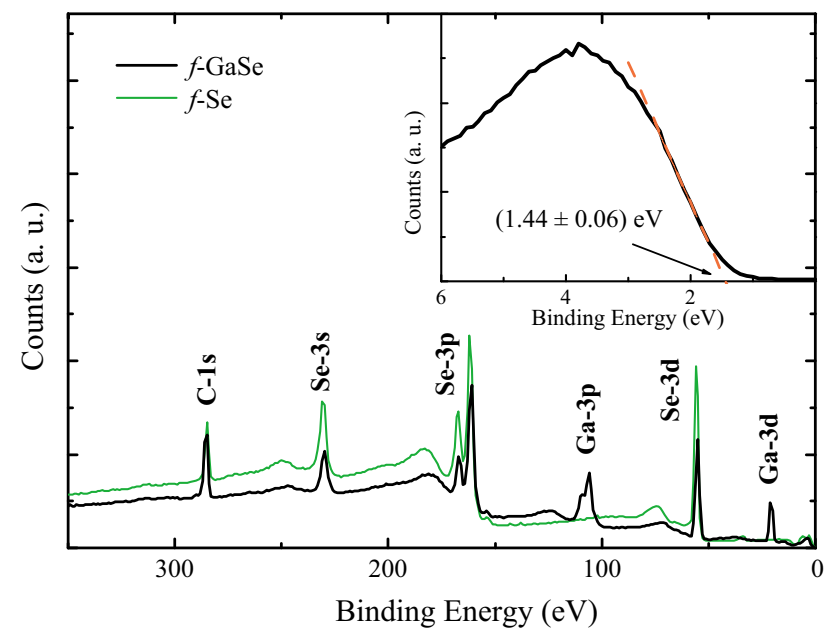

Fig. 1 Typical XPS spectrum of $f$-GaSe (black lines) and $f$-Se (green line), both deposited on $n$-Si. The inset presents the details of the valence band region of $f$-GaSe, and the fit to obtain the binding energy of the top of the valence band (dashed red line) (Color figure online)
Figure 1 also shows the $1 s$ level of carbon, at about $285 \mathrm{eV}$, which is the same position found for pure $\mathrm{C}$ in the literature [25]. This indicates that there is no need for corrections associated with sample charging effects. This peak comes from the carbon tape used to fix the samples in the sample holder. Peaks associated with other chemical species are absent, so there is no contamination in the films nor in the powders by elements such as iron, which could hypothetically be introduced during the milling process.

\subsection{Transmittance spectroscopy measurements}

Figure 2 shows the transmittance spectra of $f$-GaSe deposited on quartz (full black line) and of the bare quartz substrate (red dashed line). The fringes are due to interference effects in the film, occuring for wavelengths greater than $700 \mathrm{~nm}$. The absorption edge appears around $600 \mathrm{~nm}$, where there is a high decrease in the transmittance of $f$ GaSe. The absorption band around $2726 \mathrm{~nm}$ is due to the quartz substrate.

From the transmittance measurements, the absorption coefficient was obtained using of the Cisneros method [17], and from the corresponding values the analysis of the absorption edge was performed using Eq. 1, as described in Sect. 2.1. Figure 3 displays the absorption edges of $f$-GaSe deposited on quartz, using $m=\frac{1}{2}$ (top) and $m=2$ (bottom).

The determined value of the bandgap corresponding to the amorphous phase (Tauc's procedure) was $E_{g}^{\text {Tauc }}=$ $1.93 \pm 0.03 \mathrm{eV}$. An extended linear region was also observed in the plot equivalent to the direct allowed transitions $(m=2)$. The resultant (direct) bandgap value was $E_{g}^{d}=2.15 \pm 0.03 \mathrm{eV}$. These values can be compared with other results found in the literature. Considering only amorphous films, Mustafa et al. [8] found indirect allowed

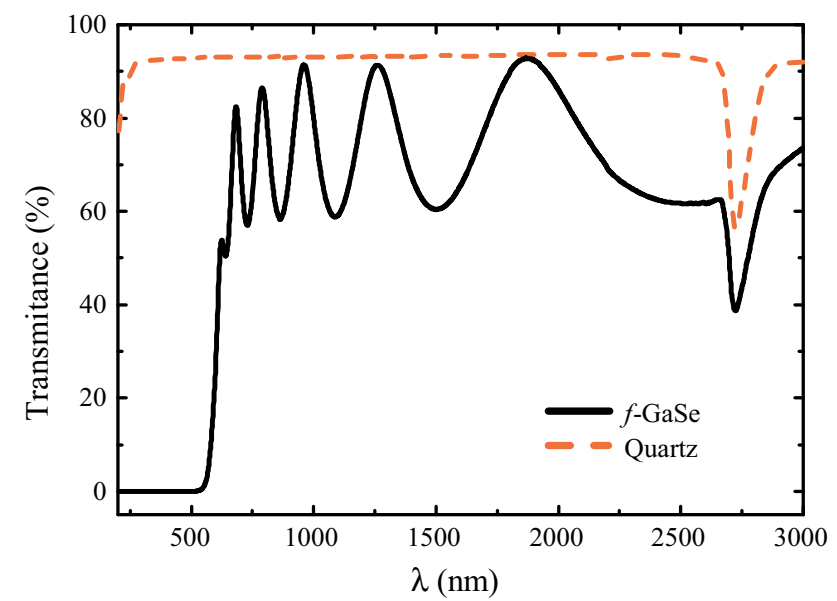

Fig. 2 Transmittance spectra of $f$-GaSe deposited on quartz (full black line) and of the bare quartz substrate (red dashed line) (Color figure online) 


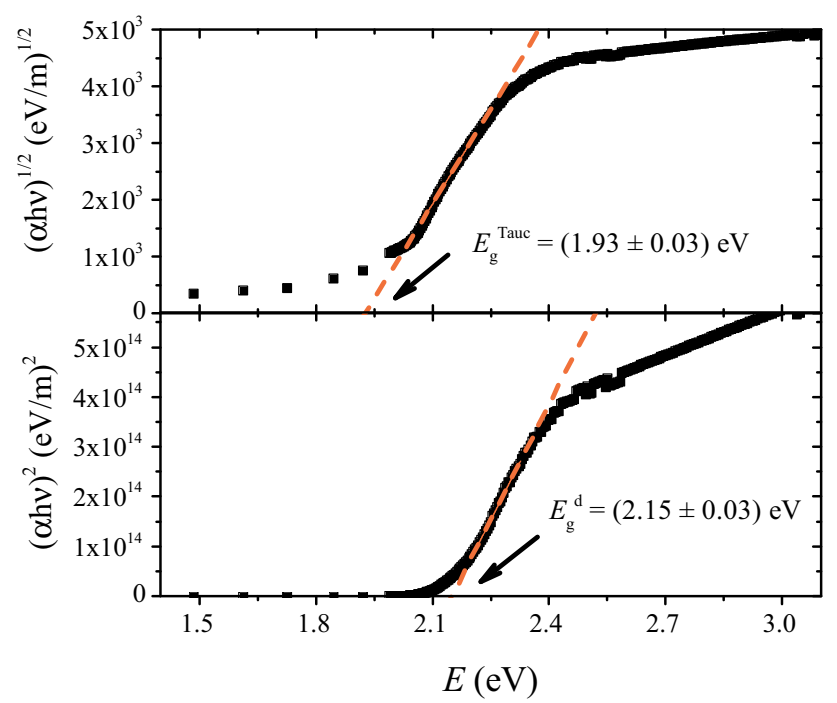

Fig. 3 Plot of $(\alpha h v)^{m} \times h v$ (black squares) and linear fit of the absorption edge given by Eq. 1 (red dashed lines) considering the Tauc's plot $\left(m=\frac{1}{2}\right.$, top $)$ and direct allowed transitions $(m=2$, bottom) for $f$-GaSe deposited on quartz (Color figure online)

gaps with values $E_{g}^{i}=1.06 \mathrm{eV}$, for $f-\mathrm{Ga}_{3} \mathrm{Se}_{2}, \quad E_{g}^{i}=$ $1.435 \mathrm{eV}$, for $f$-GaSe, and $E_{g}^{i}=2.05 \mathrm{eV}$, for $f-\mathrm{Ga}_{2} \mathrm{Se}_{3}$. For $f$-GaSe, Tripathi et al. Tripathi et al. [7] found $E_{g}^{i}=$ $(1.34 \pm 0.01) \mathrm{eV}$, and Thamilselvan et al. [11] found $E_{g}^{i}=1.44 \mathrm{eV}$. For $f-\mathrm{Ga}_{2} \mathrm{Se}_{3}$, Afifi et al. [13] obtained $E_{g}^{i}=2.056 \mathrm{eV}$ and $E_{g}^{d}=2.65 \mathrm{eV}$, and Matsushita et al. [10] found $E_{g}^{i}=1.96 \mathrm{eV}$ and $E_{g}^{d}=2.52 \mathrm{~V}$. All these films were prepared by VE considering bulk samples produced by melt-quenching. Our films, on the other hand, were made using powders produced by MA, and the preparation technique has a large influence on the physical properties of the samples, even for bulk ones. For instance, considering bulk samples, Souza et al. [9] found $E_{g}^{d}=1.85 \mathrm{eV}$ for crystalline $\mathrm{Ga}_{2} \mathrm{Se}_{3}$ produced by MA, and Adachi and Ozaki [12] obtained $E_{g}^{i}=1.75 \mathrm{eV}$ for amorphous $\mathrm{Ga}_{2} \mathrm{Se}_{3}$ produced by MQ.

From the transmittance measurements shown in Fig. 2 and using the routine described in Sect. 2.2 based on the transmittance maxima and minima it was possible to obtain the refractive index $n$ and the extinction coefficient $k$ in the low absorption region. These values are presented in Table 1. The thickness of $f$-GaSe deposited on quartz obtained in this procedure was $t=774 \pm 25 \mathrm{~nm}$.

The values of the refractive index shown in Table 1 were used in the Wemple and DiDomenico single oscillator model [18]. Figure 4 shows the plot of $\left(n^{2}-1\right)^{-1} \times E^{2}$ and its linear fit considering Eq. (2). The values found for the dispersive and oscillator energies were $E_{d}=18.6 \pm 0.4 \mathrm{eV}$ and $E_{0}=4.00 \pm 0.09 \mathrm{eV}$, respectively. Mustafa et al. [8] also determined the values of $E_{0}$ and $E_{d}$ for amorphous $\mathrm{Ga}_{x} \mathrm{Se}_{y}$
Table 1 Refractive index and extinction coefficient corresponding to the maxima and minima of the transmittance of $f$-GaSe deposited on quartz. The calculated thickness was $t=774 \pm 15 \mathrm{~nm}$

\begin{tabular}{lll}
\hline$\lambda(\mathrm{nm})$ & $n$ & $k$ \\
\hline 1989 & 2.40 & 0.00116 \\
1599 & 2.41 & 0.00184 \\
1339 & 2.42 & 0.00251 \\
1157 & 2.44 & 0.00251 \\
1019 & 2.46 & 0.00251 \\
913 & 2.48 & 0.00390 \\
834 & 2.52 & 0.00530 \\
768 & 2.55 & 0.00605 \\
719 & 2.60 & 0.00681 \\
672 & 2.64 & 0.00340 \\
\hline
\end{tabular}

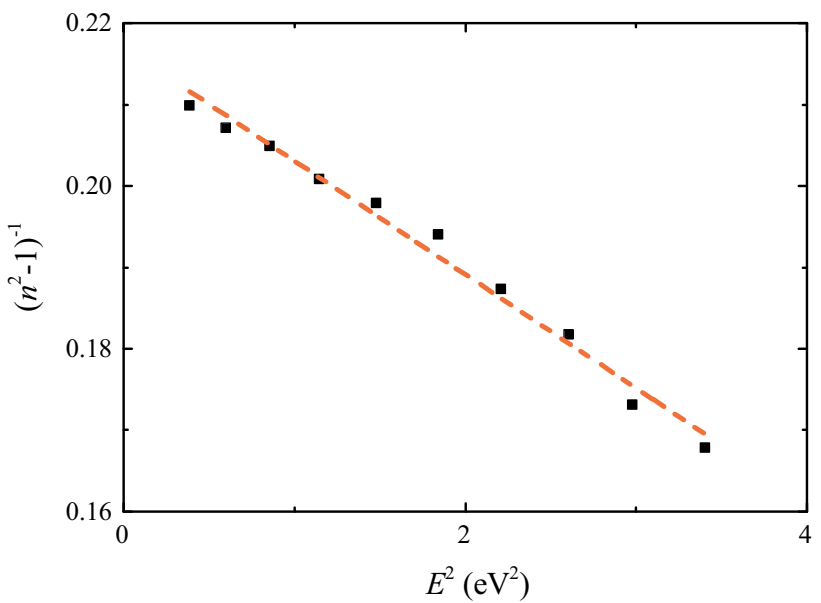

Fig. 4 Plot of $\left(n^{2}-1\right)^{-1} \times E^{2}$ for the determination of the oscillator $\left(E_{0}\right)$ and dispersive $\left(E_{d}\right)$ energies of the Wemple and DiDomenico single oscillator model [18] (black squares) and this fit considering Eq. 2 (dashed red line), for $f$-GaSe deposited on quartz (Color figure online)

thin films. They found $E_{0}=1.63 \mathrm{eV}$ and $E_{d}=9.33 \mathrm{eV}$, for $f-\mathrm{Ga}_{3} \mathrm{Se}_{2}, E_{0}=2.58 \mathrm{eV}$ and $E_{d}=12.22 \mathrm{eV}$, for $f$-GaSe, and $E_{0}=3.33 \mathrm{eV}$ and $E_{d}=12.02 \mathrm{eV}$, for $f-\mathrm{Ga}_{2} \mathrm{Se}_{3}$. Afifi et al. [13] obtained $E_{0}=3.86 \mathrm{eV}$ and $E_{d}=19.3 \mathrm{eV}$ for $f-\mathrm{Ga}_{2} \mathrm{Se}_{3}$. It is interesting to note that the relation $E_{0} \approx 2.0 E_{g}$ [26] is verified in our case.

Using the values of $E_{0}$ and $E_{d}$ obtained in the low absorption region, the values of $n$ were extrapolated to the high absorption region. The extrapolated $n$ and the measured transmittances were used to determine the absorption and extinction coefficients in the high absorption region. The $n$ and $k$ values are shown in Fig. 5. Equation 2 also allows for the determination of the static refractive index as $n^{2}(0)=1+E_{d} / E_{0}$ and the static dielectric constant $\varepsilon(0)=n^{2}(0)$. The values found were $n(0)=2.38$ and $\varepsilon(0)=5.65$, respectively.

From the values obtained for the direct optical gap $\left(E_{g}^{d}=2.15 \pm 0.03 \mathrm{eV}\right)$ and for the valence band $\left(E_{v b}=5.84 \pm 0.06 \mathrm{eV}\right)$, it is possible to estimate the value 


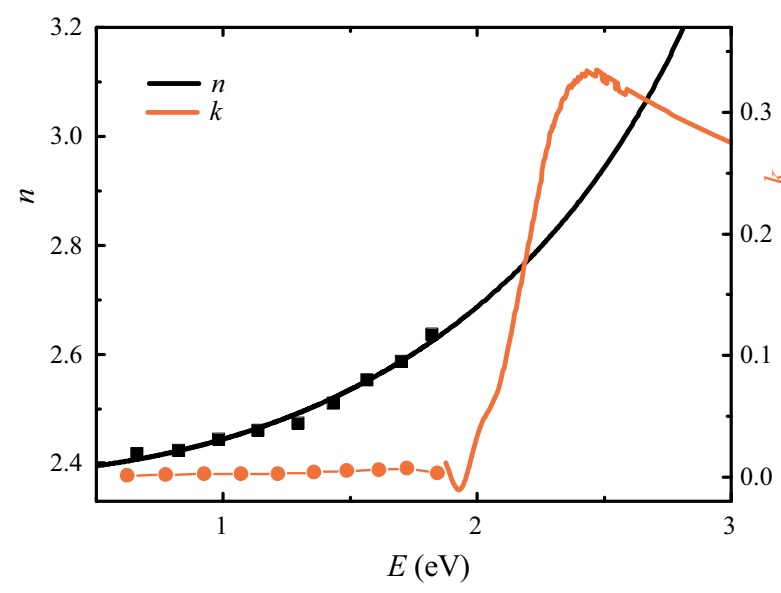

Fig. 5 Refractive index (black line and squares) and extinction coefficient (orange line and circles) for $f$-GaSe deposited on quartz. The refractive index values obtained in the low absorption region (squares) were extrapolated to higher energies using the Wemple and DiDomenico model [18]. The values of the extinction coefficient in the low absorption (circles) were calculated using the $n$ values determined on the transmittance extrema, while the values corresponding to higher energies (continuous orange line) were determined using the extrapolated $n$ values and the experimental transmittance (Color figure online)

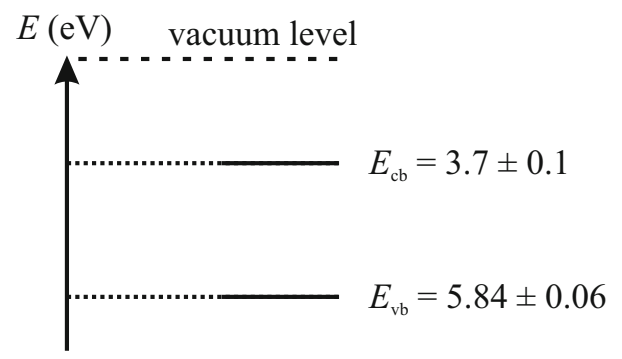

Fig. 6 Energy level diagram proposed for $f$-GaSe

of the conduction band as $E_{c b}=3.7 \pm 0.1 \mathrm{eV}$. These values allowed us to construct an energy level diagram for $f$ $\mathrm{GaSe}_{9}$, which is shown in Fig. 6. This diagram is important for the development devices based on $f$-GaSe, which are currently under investigation.

\section{Conclusion}

The electronic and optical properties of amorphous GaSe thin films produced by vacuum evaporation were investigated. The evaporation materials were $\mathrm{GaSe}_{9}$ powders produced by mechanical alloying. XPS results allow determining the valence band level, and show that there is a charge transfer between Se and $\mathrm{Ga}$ atoms and absence of contamination by other chemical species.

Transmittance spectroscopy allowed the determination of the optical gap, the refractive index, and extinction coefficient in the low and high absorption regions. In addition, it was possible to propose an energy level diagram for $f$-GaSe, which is important in order to develop applications for this alloy. All results also show that $f$-GaSe is a promising material to be used in electronic devices.

Acknowledgments We would like to thank the Brazilian agencies CNPq, CAPES and FAPESP for financial support, and LNLS for financial and technical supports (proposal SXS-10976).

\section{References}

1. S.M. Sze, K. Ng, Physics of Semiconductor Devices (Wiley, Hoboken, 2007)

2. J.P.M. Serbena, K.D. Machado, M.C. Siqueira, I.A. Hümmelgen, R.J.O. Mossanek, G.B. de Souza, J.H.D. da Silva, J. Phys. D: Appl. Phys. 47, 015304 (2014)

3. V.G. Voevodin, O.V. Voevodina, S.A. Bereznaya, Z.V. Korotchenko, A.N. Moronov, S.Y. Sarkisov, N.C. Fernelius, J.T. Goldstein, Opt. Mater. 26, 495 (2004)

4. G.B. Abdullaev, L.A. Kulevskǐ, A.M. Prokhorov, A.D. Savel'ev, E.Y. Salaev, V.V. Smirnov, JETP Lett. 16, 90 (1972)

5. K.R. Allakhverdiev, M.O. Yetis, S. Özbek, T.K. Baykara, E.Y. Salaev, Laser Phys. 19, 1092 (2009)

6. E. Cuculescu, I. Evtodiev, M. Caraman, G.G. Rusu, J. Optoelectron. Adv. Mater. 8, 119 (2006)

7. S.K. Tripathi, S. Gupta, F.I. Mustafa, N. Goyal, G.S.S. Saini, J. Phys. D: Appl. Phys. 42, 185404 (2009)

8. F.I. Mustafa, S. Gupta, N. Goyal, S.K. Tripathi, Phys. Status Solidi C 6, S135 (2009)

9. S.M. Souza, C.E.M. Campos, J.C. de Lima, T.A. Grandi, P.S. Pizani, Solid State Commun. 139, 70 (2006)

10. T. Matsushita, A. Suzuki, M. Okuda, T.T. Naug, Jpn. J. Appl. Phys. 16, 2057 (1977)

11. M. Thamilselvan, K. Premnazeer, D. Mangalaraj, S.K. Narayandass, K. Kim, J. Yi, Mater. Sci. Semicond. Process. 7, 69 (2004)

12. S. Adachi, S. Ozaki, Jpn. J. Appl. Phys. 32, 4446 (1993)

13. M.A. Afifi, A.E. Bekheet, H.T. El-Shair, I.T. Zedan, Phys. B 325, $308(2003)$

14. M.C. Siqueira, R.N.A. Maia, R.M.T. Araujo, K.D. Machado, S.F. Stolf, J. Chem. Phys. 142, 054504 (2015)

15. M.C. Siqueira, R.N.A. Maia, R.M.T. Araujo, K.D. Machado, S.F. Stolf, J.C. de Lima, C.M. Poffo, J. Appl. Phys. 116, 083514 (2014)

16. T.P. McLean, Prog. Semicond. 5, 55 (1960)

17. J.I. Cisneros, Appl. Opt. 37, 5762 (1998)

18. S.H. Wemple, M. DiDomenico, Phys. Rev. B 3, 1338 (1971)

19. F. Abelès, Optical Properties of Solids (Elsevier, North-Holland, 1972)

20. J.I. Pankove, Optical Processes in Semiconductors (Dover, New York, 1971)

21. J. Tauc, R. Grigorov, A. Vancu, Phys. Status Solidi 15, 627 (1966)

22. N.F. Mott, E.A. Davis, Electronic Processes in Non-Crystalline solids (Pergamon Press, New York, 1971)

23. S. Ambika, P.B. Barman, Phys. B 405, 822 (2010)

24. S. Tanuma, C.J. Powell, D.R. Penn, Surf. Interface Anal. 43, 689 (2011)

25. S. Hufner, Photoelectron Spectroscopy-Principles and Applicatons (Springer, Washington, 1996)

26. K. Tanaka, Thin Solid Films 66, 271 (1980) 